\title{
Anaesthetic Management of a Neonate with an Intrapericardial Teratoma: A Case Report
}

\author{
Ying Lu-Boettcher ${ }^{1}$, Prabhat Koppera ${ }^{2}$, and Lori Riegger ${ }^{2}$ \\ ${ }^{1}$ University of Wisconsin-Madison \\ ${ }^{2}$ University of Michigan Health System
}

August 6, 2020

\begin{abstract}
We describe the anaesthetic management of a 4-day-old premature infant presenting for urgent resection of a massive posterior intrapericardial teratoma. Anaesthetic challenges include anticipating cardiopulmonary collapse upon induction and hemodynamic instability associated with blood loss or tumor manipulation. Premature infants present unique challenges due to patient-to-tumor size discrepancy.
\end{abstract}

\section{Introduction:}

Intrapericardial teratomas are rare primary cardiac tumors with a reported incidence of $0.003-0.14 \%$ in published literature, accounting for $15-19 \%$ of paediatric cardiac tumors. These tumors can arise from the inner lining, cardiac muscle layer, or the pericardium, and the majority in infants and children are benign $1-4$.

During fetal life, manifestations of a cardiac tumor can include congestive heart failure, hydrops, arrhythmias, and possible stillbirth. In postnatal life cardiac tumors may exert mass effect on adjacent structures leading to inflow or outflow tract obstruction, myocardial dysfunction, respiratory distress, arrhythmias, and sudden death $_{3,5}$. Surgical resection is the treatment of choice as it can definitively alleviate mass effect exerted by the tumor. Our current anaesthetic experience is predominately based on case reports. Thus, the aim of this report is to address perioperative concerns unique to neonatal intrapericardial teratomas and aid in future anaesthetic planning.

\section{Case Description:}

We present a $2 \mathrm{~kg}$ preterm female with a large prenatally diagnosed posterior intrapericardial mass concerning for teratoma, without signs of hydrops on prenatal ultrasound which confers lower morbidity and mortality 6 .

The infant was born via spontaneous vaginal delivery at $33 \mathrm{w} 6 \mathrm{~d}$ gestation in the setting of preterm labor and was successfully intubated at minute 3-4 of life. Apgars at 1 and 5 minutes of life were 3 and 7 , respectively. UVC and UAC were placed, labs obtained, prostaglandins started while awaiting formal echocardiography, and patient transferred to paediatric cardiac ICU for further management. Postnatal echo, MRA/MRI confirmed findings of a $4.3 \mathrm{~cm} \times 3.6 \mathrm{~cm} \times 3.5 \mathrm{~cm}$ mass displacing the heart within in a massive pericardial effusion. The mass was intimately related to the roof of bilateral atria, the ascending aorta, main and right pulmonary artery, and right coronary artery. There was mass effect causing compression the superior vena cava, leftward displacement of the left pulmonary artery, anterior leftward displacement of the aorta, and mild narrowing in the distal trachea, all without evidence of tamponade (Fig 1-2). Preoperative alpha-fetoprotein level was greater than $145,000 \mathrm{ng} / \mathrm{mL}$. 
On DOL2, hypotension in the cardiac ICU prompted initiation of dopamine infusion, bedside drainage of $60 \mathrm{~mL}$ of pericardial effusion with placement of a pericardial drain.

\section{Perioperative Course:}

On DOL4, 34w3d, patient was brought to OR for tumor resection. Patient had a pre-existing 3.5 uncuffed ETT, and all inpatient infusions were maintained, including TPN, dexmedetomedine $0.3 \mathrm{mcg} / \mathrm{kg} / \mathrm{hr}$, morphine $20 \mathrm{mcg} / \mathrm{kg} / \mathrm{hr}$, and dopamine $2.5 \mathrm{mcg} / \mathrm{kg} /$ hour. Patient was given additional $0.4 \mathrm{mg}$ of midazolam and $5 \mathrm{mg}$ of rocuronium prior to transport. In addition to existing UAC and UVC, two $22 \mathrm{~g}$ IV's were established in OR for access. ETT was exchanged to 3.0 cuffed ETT uneventfully under direct laryngoscopy due to leak.

General anaesthesia was maintained with oxygen/air, sevoflurane, $5 \mathrm{mcg}$ increments of fentanyl, and 2 $\mathrm{mg}$ of rocuronium intermittently. In addition to arterial waveform and CVP, near-infrared spectroscopy (NIRS), capnography, temperature, and pulse oximetry monitoring were utilized. Via median sternotomy, with cardiopulmonary bypass on standby, the pericardium was opened and a multiloculated hemorrhagicappearing mass measuring approximately 5 centimeters in length was revealed, densely adherent along the entire lateral aspect of the ascending aorta as well as the medial aspect of the SVC. There were multiple bouts of hemodynamic instability while the mass was being resected due to the proximity of the mass to the systemic venous connections. As a result, the patient remained on dopamine throughout the case and received liberal fluid resuscitation $(25 \mathrm{~mL} / \mathrm{kg}$ of crystalloid) as well as $40 \mathrm{~mL} / \mathrm{kg}$ of pRBCs. Permissive hypotension was allowed to facilitate resection. The case did not require cardiopulmonary bypass and the chest was closed uneventfully after resection (Fig 3). She was brought to cardiac ICU intubated post operatively, and required inotropic support until post-operative day 1 . She was extubated in the intensive care unit on post-operative day 2. The patient was discharged home on post-operative day 4.

\section{Discussion:}

Anaesthetic management of an intrapericardial tumor can present a serious challenge for the anaesthesiologist. Our current anaesthetic experience is predominately based on case reports. Teratomas can be associated with mass effect and pericardial effusions resulting in cardiac compression and respiratory distress after birth. After diagnosis, the typical treatment of choice is surgical resection.

For preoperative planning, it is important to obtain two-dimensional echocardiography as well as magnetic resonance imaging for larger tumors to elucidate the tumor's involvement with adjacent structures. Determining the ability to separate the mass from vital structures, such as the great vessels and airway, is essential for anaesthetic and surgical planning. In our patient's case, MRI/MRA as well as 2-Dimensional echo were all obtained during preoperative planning.

In patients with mass effect on the respiratory tree, the goal of preventing respiratory collapse is achieved by performing intubation while the patient is ventilating spontaneously. Depending on location of tumor, airway obstruction and hemodynamic collapse may still occur, which may need to be alleviated by immediate removal/lifting up of tumor by the surgeon after sternotomy. Cardiopulmonary bypass is often on standby, either as a rescue measure or to facilitate resection. In patients with critical positional symptoms of cardiopulmonary compression, induction of anaesthesia with maintenance of spontaneous ventilation may still be unsafe. Alternatives such as extracorporeal membrane oxygenation may need to be established prior to surgical resection 7,8 . In our patient's case, mass effect on the cardiorespiratory system was somewhat mitigated after drainage of pericardial effusion. There was only a mild compression of distal trachea and we had the knowledge that she was previously uneventfully intubated during neonatal resuscitation. In our patient's case, tumor resection occurred $>34$ weeks gestational age and weight $>2000$ grams to allow for cardiopulmonary bypass rescue or ECMO 9, 10, which may be precluded in smaller patients.

Intraoperatively, hemodynamic disturbances should be anticipated with tumor manipulation and surgical blood loss. Adequate IV access is essential as hemodynamic instability is expected and significant blood loss, while rare, can occur. In our patient, an infusion of dopamine and liberal fluid resuscitation including blood products were required to maintain hemodynamic stability. In addition, epinephrine and vasopressin 
infusions were immediately available. Despite these precautions, permissive hypotension was still required to facilitate surgical resection.

Postoperatively, most patients experience smooth recovery. Our patient was uneventfully discharged on POD 4. Histological examination showed a predominately mature teratoma with immature components, with elements from all three primitive germ cell layers. Patient returned on POD 36 for a repeat cardiac MRI, which showed no residual tumor.

\section{Conclusion:}

Surgical resection remains the treatment of choice for intrapericardial teratomas. During anaesthetic management, one should anticipate cardiopulmonary collapse upon induction, particularly with non-intubated patient with severe mass effect, as well as hemodynamic instability associated with blood loss and tumor manipulation. Understanding the potential complications associated with intrapericardial teratomas was paramount in the successful anaesthetic management of our patient.

\section{Disclosure of Authors:}

1. Ying E. Lu-Boettcher, MD: None

2. Prabhat Koppera, MD: None

3. Lori Q. Riegger, MD: None

\section{Conflict of Interest:}

1. Ying E. Lu-Boettcher, MD: None

2. Prabhat Koppera, MD: None

3. Lori Q. Riegger, MD: None

\section{Author Contributions:}

1. Ying E. Lu-Boettcher: This author was clinically involved, wrote the manuscript and obtained consent.

2. Prabhat Koppera: This author was clinically involved with the case and edited the manuscript.

3. Lori Q. Riegger: This author helped with the writing, editing, and obtaining consent for the manuscript.

\section{References}

1. Malay J, Madhavi N, Satyavani A, Nishanth P, Manikyamba D. Intrapericardial Immature Teratoma with Successful Treatment in a Neonate. Indian J Pediatr. 2014; 81:1099-1101.

2. Kumar IH, Shrote V, Kumar H. Anaesthetic management of a child presenting with intrapericardial teratoma compressing the airway and the heart. Ann Card Anaesth 2009;12:63-6

3. MacKenzie S, Loken S, Kalia N, et al. Intrapericardial teratoma in the perinatal period: case report and review of the literature. J Pediatr Surg. 2005;40:13-18.

4. Uzun O, Wilson DG, Vujanic, GM, Parsons JM, De Giovanni J. Cardiac Tumours in Children. Orphanet J Rare Dis. 2007;2:11.

5. Azzakhmam M, Kessab A, Malihy A, Rouas L, Lamalmi N. Intracardiac Teratoma in an Infant: Report of a New Case and Literature Review. Case Rep Pathol. 2018; 2018: 6805234.

6. Isaacs H. Fetal Hydrops Associated with Tumors. Am J Perinatol 2008; 25: 43-68.

7. Marianeschi SM, Seddio F, Abella RF, Colagrande L, Lorio FS, Marcelletti CF. Intrapericardial teratoma in a newborn: A case report. J Card Surg 1999; 14:169-71.

8. Slinger PA, Karsli CB. Management of the patient with a large anterior mediastinal mass: Recurring myths. Thorac Anaesth Curr Opin Anaesthesiol 2007;20:1-3.

9. Cilley RE, Zwischenberger JB, Andrews AF, Bowerman RA, Roloff DW, Bartlett RH. Intracranial hemorrhage during extracorporeal membrane oxygenation in neonates. Pediatrics 1986;78(4):699-704.

10. Bui KC, LaClair P, Vanderkerhove J, Bartlett RH. ECMO in premature infants: Review of factors associated with mortality. ASAIO Trans 1991;37:54-59. 

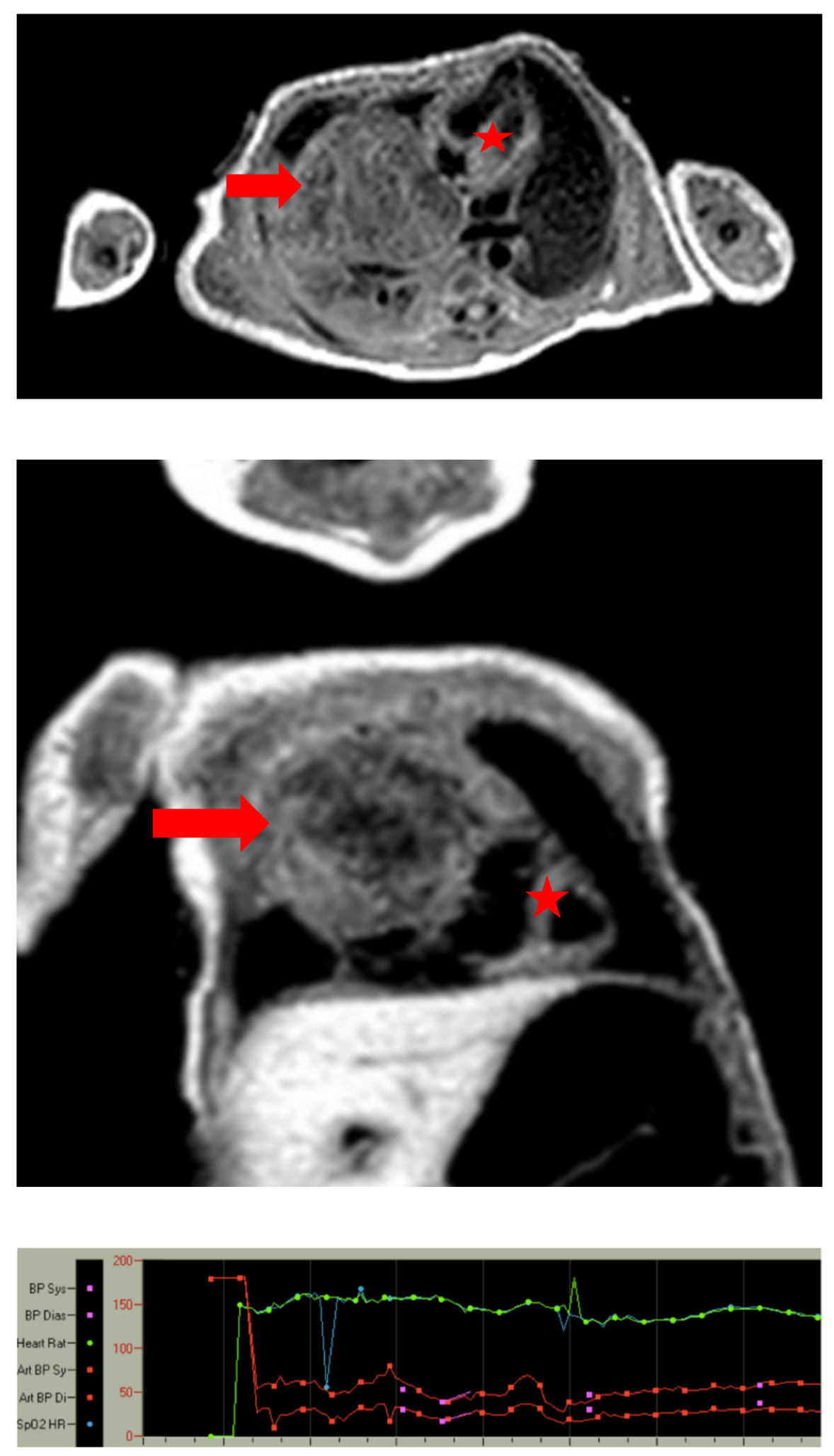